Mathematical Modelling and Analysis

Volume 21 Number 4, July 2016, 466-477

http://dx.doi.org/10.3846/13926292.2016.1183240

(c) Vilnius Gediminas Technical University, 2016
Publisher: Taylor\&Francis and VGTU

http://www.tandfonline.com/TMMA

ISSN: $1392-6292$

eISSN: $1648-3510$

\title{
Convergence Order of the Reproducing Kernel Method for Solving Boundary Value Problems*
}

\author{
Zhihong Zhao ${ }^{a}$, Yingzhen Lin ${ }^{a}$ and Jing $\mathrm{Niu}^{b}$ \\ ${ }^{a}$ School of Science, Beijing Institute of Technology \\ 519088 Zhuhai, China \\ ${ }^{b}$ School of Mathematics and Sciences, Harbin Normal University \\ 150025 Harbin, China \\ E-mail(corresp.): njirwin@163.com
}

Received April 21, 2015; revised April 22, 2015; published online July 1, 2016

\begin{abstract}
In this paper, convergence rate of the reproducing kernel method for solving boundary value problems is studied. The equivalence of two reproducing kernel spaces and some results of adjoint operator are proved. Based on the classical properties of piecewise linear interpolating function, we provide the convergence rate analysis of at least second order. Moreover, some numerical examples showing the accuracy of the proposed estimations are also given.
\end{abstract}

Keywords: reproducing kernel method, convergence analysis, boundary value problem.

AMS Subject Classification: 34G10; 68W40; 65L10.

\section{Introduction}

Boundary value problems arise in a variety of applied mathematics and physics. The numerical methods for solving the problems have attracted much attention (see [12] and [3]). In recent years, the reproducing kernel method has been proposed and applied to solve the initial boundary value problems $[2,6,7,8$, 11, 19,24]. The method has the following advantages: rigorous theory, simple process and easy to implement on computer. Especially, when we construct the reproducing kernel space in which each function satisfies boundary conditions of considered problems, we do not need to reconsider such boundary conditions in the algorithm. Therefore, it is convenient to solve the corresponding problems $[13,14,15,16,17,18,20]$.

\footnotetext{
* Correspondence to: Jing Niu, School of Mathematics and Sciences, Harbin Normal University, Harbin, 150025, China. This work was supported by the TianYuan Special Funds of the National Natural Science Foundation of China (Grant No.11426079) and Youth Foundation of Heilongjiang Province(Grant No.JJ2016QN0211) and National Natural Science Funds of China(Grant No.11401145) and Science Research Foundation of Heilongjiang Province (Grant No. A201411).
} 
The uniform convergence of the algorithm has been discussed in [22] and [23]. The numerical complexity of the algorithm in [10] is three polynomialtime. Research about convergence order of the reproducing kernel method is important to solve practical problems. In the range of allowable error, convergence order directly affects the computing speed of the algorithm as solving practical problems. Motivated by all the works above, we will complete the necessary work about convergence order of the reproducing kernel method in this paper. It is worthy to note that our approach is better than the previous reproducing kernel space method because we avoid the complex process of orthogonal.

The rest of the paper is organized as follows: in the next section, we introduce the reproducing kernel method for solving problems and provide some necessary Lemmas. Then we present the some prepared theorems in Section 3. Error order estimation is analyzed in Section 4. In Section 5, some examples are given to show our main conclusion. Section 6 ends this paper with a brief conclusion.

\section{The reproducing kernel method for solving the boundary value problems}

Definition 1 . The reproducing kernel space $W_{m}[0,1]$ is defined by $W_{m}[0,1]=$ $\left\{u \mid u^{(m-1)}(x)\right.$ is an absolutely continuous real value function in $[0,1], u^{(m)}(x) \in$ $\left.L^{2}[0,1]\right\}$. The inner product of $W_{m}[0,1]$ is defined by

$$
\langle u, v\rangle_{W_{m}}=\sum_{i=0}^{m-1} u^{(i)}(0) v^{(i)}(0)+\int_{0}^{1} u^{(m)}(x) v^{(m)}(x) d x .
$$

Definition 2. The reproducing kernel space $H_{m}[0,1]$ is defined by $H_{m}[0,1]=$ $\left\{u \mid u^{(m-1)}(x)\right.$ is an absolutely continuous real value function in $[0,1], u^{(m)}(x) \in$ $\left.L^{2}[0,1]\right\}$. The inner product of $H_{m}[0,1]$ is defined as follows

$$
\langle u, v\rangle_{H_{m}}=\sum_{i=0}^{m} \int_{0}^{1} u^{(i)}(x) v^{(i)}(x) d x .
$$

Let $R_{t}^{m}(x), K_{t}^{m}(x)$ be the reproducing kernel functions of $W_{m}[0,1]$ and $H_{m}[0,1]$, respectively. From the definition of the inner product of $H_{m}[0,1]$, the following Lemma 1 can be easily obtained.

Lemma 1. In space $H_{m}[0,1]$, when $i \leq j$, then

$$
\|u\|_{H_{i}} \leq\|u\|_{H_{j}} .
$$

Lemma 2. Space $W_{m}[0,1]$ is the equivalent with the space $H_{m}[0,1]$.

Proof. We need to get the conclusion: $\|u\|_{H_{m}}^{2} \leq C\|u\|_{W_{m}}^{2}$ and $\|u\|_{W_{m}}^{2} \leq$ $D\|u\|_{H_{m}}^{2}$, where $C$ and $D$ are constants. By reproducibility, we have

$$
u(t)=\left\langle u, R_{t}^{m}\right\rangle_{W_{m}}, \quad u^{(i)}(t)=\left\langle u, \partial_{t}^{i} R_{t}^{m}\right\rangle_{W_{m}}, \quad 0 \leq i \leq m-1,
$$


so

$$
\left|u^{(i)}(t)\right|=\left|\left\langle u, \partial_{t}^{i} R_{t}^{m}\right\rangle_{W_{m}}\right| \leq\|u\|_{W_{m}}\left\|\partial_{t}^{i} R_{t}^{m}\right\|_{W_{m}} \leq C_{i}\|u\|_{W_{m}},
$$

then

$$
\|u\|_{H_{m}}^{2}=\int_{0}^{1} \sum_{i=0}^{m}\left(u^{(i)}\right)^{2} d t \leq \int_{0}^{1} \sum_{i=0}^{m-1} C_{i}^{2}\|u\|_{W_{m}}^{2} d t+\int_{0}^{1}\left(u^{(m)}\right)^{2} d t \leq C\|u\|_{W_{m}}^{2} .
$$

On the other hand,

$$
u(t)=\left\langle u, K_{t}^{m}\right\rangle_{H_{m}}, \quad u^{(i)}(t)=\left\langle u, \partial_{t}^{i} K_{t}^{m}\right\rangle_{H_{m}}, \quad 0 \leq i \leq m-1,
$$

SO

$$
\left|u^{(i)}(0)\right|=\left|\left\langle u,\left(\partial_{t}^{i} K_{t}^{m}\right)_{t=0}\right\rangle_{H_{m}}\right| \leq\|u\|_{H_{m}}\left\|\left(\partial_{t}^{i} K_{t}^{m}\right)_{t=0}\right\|_{H_{m}} \leq D_{i}\|u\|_{H_{m}},
$$

then

$$
\begin{aligned}
\|u\|_{W_{m}}^{2} & =\sum_{i=0}^{m-1}\left(u^{(i)}(0)\right)^{2}+\int_{0}^{1}\left(u^{(m)}\right)^{2} d t \\
& \leq \sum_{i=0}^{m-1} D_{i}^{2}\|u\|_{H_{m}}^{2}+\int_{0}^{1}\left(u^{(m)}\right)^{2} d t \leq D\|u\|_{H_{m}}^{2} .
\end{aligned}
$$

From Lemmas 1 and 2, we can obtain the following Corollary.

Corollary 1.

$$
\|u\|_{W_{i}} \leq C\|u\|_{W_{j}}, \quad i \leq j .
$$

In this paper, we consider the following equations on the assumption that the solution is existent and unique.

$$
\left\{\begin{array}{l}
u^{(m-1)}(x)+a_{1}(x) u^{(m-2)}(x)+\cdots+a_{m-1}(x) u(x)=f(x), \\
T_{1} u=0, T_{2} u=0, \ldots, T_{m-1} u=0
\end{array}\right.
$$

where $a_{1}(x), \ldots, a_{m-1}(x), f(x) \in W_{1}[0,1], T_{i}(i=1, \ldots, m-1)$ are bounded linear functionals on $W_{m}[0,1]$.

In order to solve equation (2.1) by using the reproducing kernel method, we firstly construct the following reproducing kernel space

$$
W_{m}^{0}[0,1]=\left\{u \mid u \in W_{m}[0,1], T_{1} u=0, T_{2} u=0, \ldots, T_{m-1} u=0\right\} .
$$

By defining the linear operator $\mathbb{L}: W_{m}^{0}[0,1] \rightarrow W_{1}[0,1]$ as

$$
\mathbb{L} u(x)=u^{(m-1)}(x)+a_{1}(x) u^{(m-2)}(x)+\cdots+a_{m-1}(x) u(x)
$$

equation (2.1) changes to

$$
\mathbb{L} u(x)=f(x)
$$


Next we describe an improvement of the reproducing kernel method to find the approximation solution for equation (2.2). More precisely, let $R_{t}(x)$ and $r_{t}(x)$ be the reproducing kernel functions of $W_{m}^{0}[0,1]$ and $W_{1}[0,1]$, respectively. Moreover, $\psi_{i}(x)=\mathbb{L}^{*} r_{x_{i}}(x)$, where $\left\{x_{i}\right\}_{i=1}^{\infty}$ is dense set on $[0,1]$, and $\mathbb{L}^{*}$ is the adjoint operator of $\mathbb{L}$. Note that $S_{n} \triangleq \operatorname{span}\left\{\psi_{i}(x)\right\}_{1 \leq i \leq n}$, and $\mathbb{P}_{n}: W_{m}^{0} \rightarrow S_{n}$ is an orthogonal projection operator. It is easy to observe that

$$
\left\langle\mathbb{P}_{n} u, \psi_{i}\right\rangle=\left\langle u, \psi_{i}\right\rangle=(\mathbb{L} u)\left(x_{i}\right)
$$

Thus we get the following conclusion:

Corollary 2. If $u$ is the solution of equation (2.2), then $u_{n} \triangleq \mathbb{P}_{n} u$ is the solution of $\left\langle v, \psi_{i}\right\rangle=f\left(x_{i}\right)$.

In this case, $u_{n}$ is uniformly convergent to $u$ in $[0,1]$. In fact, applying $\left\|u_{n}-u\right\| \rightarrow 0$ one obtains the following uniformly convergence:

$$
\left|u_{n}(x)-u(x)\right|=\left|\left\langle u_{n}-u, R_{x}\right\rangle\right| \leq|| u_{n}-u\left|\left\||| R_{x}\right\| \rightarrow 0 .\right.
$$

Therefore we introduce specific algorithm. Sine $u_{n} \in S_{n}$, indeed the formula is

$$
u_{n}=\sum_{i=1}^{n} \alpha_{i} \psi_{i}
$$

In this way we just search $\alpha_{i}$. With the help of Corollary 2 we now obtain that

$$
f\left(x_{j}\right)=\left\langle u_{n}, \psi_{j}\right\rangle=\sum_{i=1}^{n} \alpha_{i}\left\langle\psi_{i}, \psi_{j}\right\rangle, j=1,2, \ldots, n
$$

and set matrices

$G=\left(\left\langle\psi_{i}, \psi_{j}\right\rangle\right)_{1 \leq i, j \leq n}, \vec{\alpha}=\left(\alpha_{1}, \alpha_{2}, \ldots, \alpha_{n}\right)^{\mathrm{T}}, \vec{F}=\left(f\left(x_{1}\right), f\left(x_{2}\right), \ldots, f\left(x_{n}\right)\right)^{\mathrm{T}}$.

It follows that $\vec{\alpha}=G^{-1} \vec{F}$. Then the approximate solution $u_{n}$ is obtained.

\section{Several prepared theorems}

For convenience, we only discuss convergence order for the case $m=5$ in equation (2.1) by the reproducing kernel method. The case is completely similar for the other value of $m$. Suppose that $a_{1}(x), \ldots, a_{4}(x), f(x) \in W_{2}[0,1] \subset$ $W_{1}[0,1]$, we have the following theorems:

\section{Theorem 1.}

$$
W_{5}^{0}[0,1]=\operatorname{Im} \mathbb{L}^{*}, \quad\left(k e r \mathbb{L}^{*}\right)^{\perp}=\operatorname{Im} \mathbb{L}=W_{1}[0,1] .
$$

Proof. For any $u \in\left(I m \mathbb{L}^{*}\right)^{\perp}$, since $\psi_{i}(x)=\mathbb{L}^{*} r_{x_{i}}(x) \in I m \mathbb{L}^{*}$, we get

$$
\left\langle u, \psi_{i}(x)\right\rangle_{W_{5}}=0 \text {. }
$$


That is

$$
0=\left\langle u, \psi_{i}(x)\right\rangle_{W_{5}}=\left\langle\mathbb{L} u, r_{x_{i}}\right\rangle_{W_{1}}=\mathbb{L} u\left(x_{i}\right) .
$$

Noting that $\left\{x_{i}\right\}_{i=1}^{\infty}$ is dense set in $[0,1]$, therefore $\mathbb{L} u=0$. It follows that $u=0$ from the existence of $\mathbb{L}^{-1}$. This implies $\left(\operatorname{Im} \mathbb{L}^{*}\right)^{\perp}=\{0\}$. Hence $\operatorname{Im} \mathbb{L}^{*}=$ $W_{5}^{0}[0,1]$. Likewise, we can prove $\left(k e r \mathbb{L}^{*}\right)^{\perp}=\operatorname{Im} \mathbb{L}=W_{1}[0,1]$.

Lemma 3. $u \in W_{6}$ if and only if $\mathbb{L} u \in W_{2}$.

Proof. If $u \in W_{6}$, from the definition of the operator $\mathbb{L}$ and assumption of functions $a_{1}(x), a_{2}(x), a_{3}(x), a_{4}(x)$, we deduce that $\mathbb{L} u \in W_{2}$. Now, assume that $\mathbb{L} u \in W_{2}$. Then we derive $u \in W_{6}$. Otherwise, if $u \notin W_{6}$, then in the light of Definition 1, we deduce $u^{(5)}$ is not an absolutely continuous function or $u^{(6)} \notin L^{2}[0,1]$. Obviously, $(\mathbb{L} u)^{\prime}$ is not absolutely continuous or $(\mathbb{L} u)^{\prime \prime} \notin$ $L^{2}[0,1]$. It contradicts with $\mathbb{L} u \in W_{2}$.

Lemma 4. $w \in W_{2}$ if and only if $\mathbb{L}^{*} w \in W_{6}$.

Proof. By the reproducing kernel method, we have

$$
\begin{aligned}
\mathbb{L}^{*} w(t)=\left\langle\mathbb{L}^{*} w, R_{t}\right\rangle_{W_{5}}=\left\langle w, \mathbb{L} R_{t}\right\rangle_{W_{1}} \\
=w(0) \mathbb{L} R_{t}(0)+\int_{0}^{1} w^{\prime} \partial_{x} \mathbb{L} R_{t}(x) d x \\
=w(0)\left[\partial_{x}^{4} R_{t}(0)+a_{1}(0) \partial_{x}^{3} R_{t}(0)+a_{2}(0) \partial_{x}^{2} R_{t}(0)+a_{3}(0) \partial_{x} R_{t}(0)\right. \\
\left.\quad+a_{4}(0) R_{t}(0)\right]+\int_{0}^{1} w^{\prime}\left[\partial_{x}^{5} R_{t}(x)+a_{1} \partial_{x}^{4} R_{t}(x)+\left(a_{1}^{\prime}+a_{2}\right) \partial_{x}^{3} R_{t}(x)\right. \\
\left.\quad+\left(a_{2}^{\prime}+a_{3}\right) \partial_{x}^{2} R_{t}(x)+\left(a_{3}^{\prime}+a_{4}\right) \partial_{x} R_{t}(x)+a_{4}^{\prime} R_{t}(x)\right] d x
\end{aligned}
$$

Considering the symmetry of the producing kernel function $R_{t}(x)$ and its piecewise continuity on $t$, we know from [21] $\partial_{t}^{j} \partial_{x}^{i} R_{t}(x)$ is continuous function as $0 \leq i+j \leq 8 ; \partial_{t}^{j} \partial_{x}^{i} R_{t}(x)$ is jump discontinuous on $t$ as $i+j=9 ; \partial_{t}^{j} \partial_{x}^{i} R_{t}(x)=$ $\delta(x-t)$ as $i+j=10$. Hence, we have

$$
\begin{array}{rl}
\partial_{t}^{5} \mathbb{L}^{*} & w(t)=w(0)\left[\partial_{t}^{5} \partial_{x}^{4} R_{t}(0)+a_{1}(0) \partial_{t}^{5} \partial_{x}^{3} R_{t}(0)+a_{2}(0) \partial_{t}^{5} \partial_{x}^{2} R_{t}(0)\right. \\
& \left.+a_{3}(0) \partial_{t}^{5} \partial_{x} R_{t}(0)+a_{4}(0) \partial_{t}^{5} R_{t}(0)\right]+\int_{0}^{1} w^{\prime}\left[\partial_{t}^{5} \partial_{x}^{5} R_{t}(x)+a_{1} \partial_{t}^{5} \partial_{x}^{4} R_{t}(x)\right. \\
& +\left(a_{1}^{\prime}+a_{2}\right) \partial_{t}^{5} \partial_{x}^{3} R_{t}(x)+\left(a_{2}^{\prime}+a_{3}\right) \partial_{t}^{5} \partial_{x}^{2} R_{t}(x)+\left(a_{3}^{\prime}+a_{4}\right) \partial_{t}^{5} \partial_{x} R_{t}(x) \\
& \left.+a_{4}^{\prime} \partial_{t}^{5} R_{t}(x)\right] d x \\
= & w(0)\left[\partial_{t}^{5} \partial_{x}^{4} R_{t}(0)+a_{1}(0) \partial_{t}^{5} \partial_{x}^{3} R_{t}(0)+a_{2}(0) \partial_{t}^{5} \partial_{x}^{2} R_{t}(0)+a_{3}(0) \partial_{t}^{5} \partial_{x} R_{t}(0)\right. \\
& \left.+a_{4}(0) \partial_{t}^{5} R_{t}(0)\right]+\int_{0}^{1} w^{\prime}\left[\delta(x-t)+a_{1} \partial_{t}^{5} \partial_{x}^{4} R_{t}(x)+\left(a_{1}^{\prime}+a_{2}\right) \partial_{t}^{5} \partial_{x}^{3} R_{t}(x)\right. \\
& \left.+\left(a_{2}^{\prime}+a_{3}\right) \partial_{t}^{5} \partial_{x}^{2} R_{t}(x)+\left(a_{3}^{\prime}+a_{4}\right) \partial_{t}^{5} \partial_{x} R_{t}(x)+a_{4}^{\prime} \partial_{t}^{5} R_{t}(x)\right] d x \\
= & w(0)\left[\partial_{t}^{5} \partial_{x}^{4} R_{t}(0)+a_{1}(0) \partial_{t}^{5} \partial_{x}^{3} R_{t}(0)+a_{2}(0) \partial_{t}^{5} \partial_{x}^{2} R_{t}(0)+a_{3}(0) \partial_{t}^{5} \partial_{x} R_{t}(0)\right.
\end{array}
$$




$$
\begin{aligned}
& \left.+a_{4}(0) \partial_{t}^{5} R_{t}(0)\right]+w^{\prime}(t)+\int_{0}^{1} w^{\prime}\left[a_{1} \partial_{t}^{5} \partial_{x}^{4} R_{t}(x)+\left(a_{1}^{\prime}+a_{2}\right) \partial_{t}^{5} \partial_{x}^{3} R_{t}(x)\right. \\
& \left.+\left(a_{2}^{\prime}+a_{3}\right) \partial_{t}^{5} \partial_{x}^{2} R_{t}(x)+\left(a_{3}^{\prime}+a_{4}\right) \partial_{t}^{5} \partial_{x} R_{t}(x)+a_{4}^{\prime} \partial_{t}^{5} R_{t}(x)\right] d x
\end{aligned}
$$

In addition

$$
\begin{aligned}
\partial_{t}^{6} & \mathbb{L}^{*} w(t) \\
= & w(0)\left[\partial_{t}^{6} \partial_{x}^{4} R_{t}(0)+a_{1}(0) \partial_{t}^{6} \partial_{x}^{3} R_{t}(0)+a_{2}(0) \partial_{t}^{6} \partial_{x}^{2} R_{t}(0)+a_{3}(0) \partial_{t}^{6} \partial_{x} R_{t}(0)\right. \\
& \left.+a_{4}(0) \partial_{t}^{6} R_{t}(0)\right]+w^{\prime \prime}(t)+\int_{0}^{1} w^{\prime}\left[a_{1} \partial_{t}^{6} \partial_{x}^{4} R_{t}(x)+\left(a_{1}^{\prime}+a_{2}\right) \partial_{t}^{6} \partial_{x}^{3} R_{t}(x)\right. \\
& \left.+\left(a_{2}^{\prime}+a_{3}\right) \partial_{t}^{6} \partial_{x}^{2} R_{t}(x)+\left(a_{3}^{\prime}+a_{4}\right) \partial_{t}^{6} \partial_{x} R_{t}(x)+a_{4}^{\prime} \partial_{t}^{6} R_{t}(x)\right] d x \\
= & w(0)\left[\partial_{t}^{6} \partial_{x}^{4} R_{t}(0)+a_{1}(0) \partial_{t}^{6} \partial_{x}^{3} R_{t}(0)+a_{2}(0) \partial_{t}^{6} \partial_{x}^{2} R_{t}(0)+a_{3}(0) \partial_{t}^{6} \partial_{x} R_{t}(0)\right. \\
& \left.+a_{4}(0) \partial_{t}^{6} R_{t}(0)\right]+w^{\prime \prime}(t)+\int_{0}^{1} w^{\prime}\left[a_{1} \delta(x-t)+\left(a_{1}^{\prime}+a_{2}\right) \partial_{t}^{6} \partial_{x}^{3} R_{t}(x)\right. \\
& \left.+\left(a_{2}^{\prime}+a_{3}\right) \partial_{t}^{6} \partial_{x}^{2} R_{t}(x)+\left(a_{3}^{\prime}+a_{4}\right) \partial_{t}^{6} \partial_{x} R_{t}(x)+a_{4}^{\prime} \partial_{t}^{6} R_{t}(x)\right] d x \\
= & w(0)\left[\partial_{t}^{6} \partial_{x}^{4} R_{t}(0)+a_{1}(0) \partial_{t}^{6} \partial_{x}^{3} R_{t}(0)+a_{2}(0) \partial_{t}^{6} \partial_{x}^{2} R_{t}(0)+a_{3}(0) \partial_{t}^{6} \partial_{x} R_{t}(0)\right. \\
& \left.+a_{4}(0) \partial_{t}^{6} R_{t}(0)\right]+w^{\prime \prime}(t)+w^{\prime}(t) a_{1}(t)+\int_{0}^{1} w^{\prime}\left[\left(a_{1}^{\prime}+a_{2}\right) \partial_{t}^{6} \partial_{x}^{3} R_{t}(x)\right. \\
& \left.+\left(a_{2}^{\prime}+a_{3}\right) \partial_{t}^{6} \partial_{x}^{2} R_{t}(x)+\left(a_{3}^{\prime}+a_{4}\right) \partial_{t}^{6} \partial_{x} R_{t}(x)+a_{4}^{\prime} \partial_{t}^{6} R_{t}(x)\right] d x
\end{aligned}
$$

It can be seen $w^{\prime}(t)$ is absolutely continuous function in $[0,1]$. Therefore $\partial_{t}^{5} \mathbb{L}^{*} w(t)$ is absolutely continuous function in $[0,1]$. Moreover, $w^{\prime \prime}(t) \in L^{2}[0,1]$ Implies that $\partial_{t}^{6} \mathbb{L}^{*} w(t) \in L^{2}[0,1]$ and the other way is also true. Thus, we obtain $\mathbb{L}^{*} w \in W_{6}$ if and only if $w \in W_{2}$.

Theorem 2. Suppose that $u \in W_{5}^{0}$ is the solution of equation (2.2) and $u_{n}=$ $\mathbb{P} u \in S_{n}$, then

$$
\left\|u-u_{n}\right\|_{W_{5}^{0}[0,1]} \leq\left\|\mathbb{L}^{*}\right\|\left\|w-w_{n}\right\|_{W_{1}}
$$

where $w \in W_{2}, u=\mathbb{L}^{*} w$. Let $w_{n}=\mathbb{P}_{\tilde{w}_{n}} w$, where $\tilde{w}_{n}=\operatorname{span}\left\{r_{x_{i}}(t)\right\}_{i=1}^{n}$.

Proof. Since $u=\mathbb{L}^{*} w, \mathbb{L}^{*} w_{n} \in W_{5}^{0}[0,1]$, one has

$$
\left\|u-u_{n}\right\|_{W_{5}^{0}} \leq\left\|\mathbb{L}^{*} w-\mathbb{L}^{*} w_{n}\right\|_{W_{5}^{0}} \leq\left\|\mathbb{L}^{*}\right\|\left\|w-w_{n}\right\|_{W_{1}} .
$$

Theorem 3. On the same assumption of Theorem 2, we get

$$
\left|u(x)-u_{n}(x)\right| \leq\left\|u-u_{n}\right\|_{W_{5}^{0}}\left\|R_{t}-\mathbb{P}_{n} R_{t}\right\|_{W_{5}^{0}} .
$$

Proof. Because of $u, u_{n} \in W_{5}^{0}$ and $u-u_{n} \in S_{n}^{\perp}$, it can be obtained

$$
\begin{aligned}
\left|u(x)-u_{n}(x)\right| & =\left|\left\langle u-u_{n}, R_{t}\right\rangle_{W_{5}^{0}}\right|=\left|\left\langle u-u_{n}, R_{t}-\mathbb{P}_{n} R_{t}\right\rangle_{W_{5}^{0}}\right| \\
& \leq\left\|u-u_{n}\right\|_{W_{5}^{0}}\left\|R_{t}-\mathbb{P}_{n} R_{t}\right\|_{W_{5}^{0}} .
\end{aligned}
$$




\section{Error order estimation}

Lemma 5. Suppose that $u \in W_{5}^{0}[0,1]$, then there exists a constant $C$ such that

$$
\|u\|_{W_{5}^{0}} \leq C\|\mathbb{L} u\|_{W_{1}} .
$$

Proof. Otherwise, for any $n \in \mathbb{N}$, there exists $v_{n} \in W_{5}^{0}[0,1]$, so that

$$
\left\|v_{n}\right\|_{W_{5}^{0}}>n\left\|\mathbb{L} v_{n}\right\|_{W_{1}} .
$$

That is

$$
\left\|v_{n} /\right\| v_{n}\|\|_{W_{5}^{0}}>n\left\|\mathbb{L} v_{n} /\right\| v_{n}\|\|_{W_{1}} .
$$

We still mark $v_{n} /\left\|v_{n}\right\|$ as $v_{n}$, then $\left\|v_{n}\right\|_{W_{5}^{0}}=1,\left\|v_{n}\right\|_{W_{5}^{0}} / n>\left\|\mathbb{L} v_{n}\right\|_{W_{1}}$, furthermore, $\left\|\mathbb{L} v_{n}\right\|_{W_{1}} \rightarrow 0$. Meanwhile, $v_{n}$ are bounded on $W_{5}^{0}[0,1]$, so $v_{n}$ exists an convergent subsequence. The subsequence is marked as $v_{n}$. It follows that $v_{n} \rightarrow v \in W_{5}^{0}[0,1]$. Since

$$
\left|\left\|v_{n}\right\|_{W_{5}^{0}}-\|v\|_{W_{5}^{0}}\right| \leq\left\|v_{n}-v\right\|_{W_{5}^{0}},
$$

then $\|v\|_{W_{5}^{0}}=1$. In addition, since $\mathbb{L} v_{n} \rightarrow \mathbb{L} v$, then $\mathbb{L} v=0$. This implies $v=0$. It contradicts with $\|v\|_{W_{5}^{0}}=1$.

Likewise, we can prove the following Lemma 6.

Lemma 6. Suppose that $w \in W_{1} \cap W_{2}$, then there exists a constant $C$ such that

$$
\|w\|_{W_{2}} \leq C\left\|\mathbb{L}^{*} w\right\|_{W_{5}^{0}}
$$

The following statement is well known (see [1]): suppose that $g \in C^{2}[a, b]$ and $\Pi \triangle g$ is piecewise linear interpolating function of $g$. That is, $\left.\Pi \triangle g\right|_{\left[x_{i-1}, x_{i}\right]}$ is linear function, which satisfies $\Pi \triangle g\left(x_{i}\right)=g\left(x_{i}\right)$, then

$$
\|g-\Pi \triangle g\|_{L^{2}} \leq C h^{2}\left\|g^{\prime \prime}\right\|_{L^{2}}, \quad\left\|g^{\prime}-(\Pi \triangle g)^{\prime}\right\|_{L^{2}} \leq \tilde{C} h\left\|g^{\prime \prime}\right\|_{L^{2}},
$$

where $h$ is the maximum step size of points $\left\{x_{i}\right\}_{1 \leq i \leq n}$. According to the definitions of inner products of $W_{2}[0,1]$ and $H_{2}[0,1]$, applying Lemmas 1,2 and Corollary 1 , we have

$$
\|g-\Pi \triangle g\|_{L^{2}} \leq K_{1} h^{2}\|g\|_{W_{2}}, \quad\left\|g^{\prime}-(\Pi \triangle g)^{\prime}\right\|_{L^{2}} \leq K_{2} h\|g\|_{W_{2}} .
$$

Furthermore,

$$
\begin{aligned}
& \|g-\Pi \triangle g\|_{L^{2}}+\left\|g^{\prime}-(\Pi \triangle g)^{\prime}\right\|_{L^{2}} \leq K_{3} h\left\|_{g}\right\|_{W_{2}}, \\
& \|g-\Pi \triangle g\|_{H_{2}} \leq K_{4} h\|g\|_{W_{2}}, \quad\|g-\Pi \triangle g\|_{W_{2}} \leq K_{5} h\|g\|_{W_{2}} .
\end{aligned}
$$

Noting that the reproducing kernel function $r_{t}(x)=1+\frac{1}{2}[x+t-|x-t|]$, then $r_{x_{i}}(x)$ are linear function on every set $\left[x_{j-1}, x_{j}\right]$. Therefore $w_{n}(x)=\mathbb{P}_{\tilde{w}_{n}} w(x)$ 
is piecewise linear interpolating function of $w(x)$. Thus, applying (4.3) to the second factor of the right side of (3.1), we have

$$
\left\|w-w_{n}\right\|_{W_{1}} \leq C_{1}\left\|w-w_{n}\right\|_{W_{2}} \leq K h\|w\|_{W_{2}} .
$$

Applying (4.1), (4.2) to (4.4), we obtain

$$
\begin{aligned}
\left\|w-w_{n}\right\|_{W_{1}} & \leq C_{2} h\|w\|_{W_{2}} \leq C_{3} h\left\|\mathbb{L}^{*} w\right\|_{W_{5}^{0}} \\
& =C_{3} h\|u\|_{W_{5}^{0}} \leq C_{4} h\|\mathbb{L} u\|_{W_{1}}=C_{4} h\|f\|_{W_{1}} .
\end{aligned}
$$

We analyze the second factor of the right side of $(3.2):\left\|R_{t}-\mathbb{P}_{n} R_{t}\right\|_{W_{5}^{0}}$. Since $R_{t} \in W_{5}^{0}=I m \mathbb{L}^{*}$, then $R_{t}=\mathbb{L}^{*} \tilde{w}$. Owing to the fourth-order smoothness of the reproducing kernel function $R_{t}$, we get $\tilde{w} \in W_{2}$.

Let $\tilde{w}_{n}=\mathbb{P}_{\tilde{w}_{n}} \tilde{w}$, then we have

$$
\begin{aligned}
\left\|R_{t}-\mathbb{P}_{n} R_{t}\right\|_{W_{5}^{0}} & \leq\left\|\mathbb{L}^{*} \tilde{w}-\mathbb{L}^{*} \tilde{w}_{n}\right\|_{W_{5}^{0}} \leq\left\|\mathbb{L}^{*}\right\|\left\|\tilde{w}-\tilde{w}_{n}\right\|_{W_{1}} \leq D_{1}\left\|\tilde{w}-\tilde{w}_{n}\right\|_{W_{2}} \\
& \leq D_{2} h\|\tilde{w}\|_{W_{2}} \leq D_{3} h\left\|\mathbb{L}^{*} \tilde{w}\right\|_{W_{5}^{0}}=D_{3} h\left\|R_{t}\right\|_{W_{5}^{0}}
\end{aligned}
$$

Summarizing (3.1), (3.2), (4.4), (4.5), we have

$$
\left|u-u_{n}\right| \leq C_{4} h\|f\|_{W_{1}} D_{3} h\left\|R_{t}\right\|_{W_{5}^{0}}=C h^{2}\|f\|_{W_{1}}\left\|R_{t}\right\|_{W_{5}^{0}} \leq M h^{2}\|f\|_{W_{1}} .
$$

According to the above discussion, we obtain the following main conclusions.

Theorem 4. The approximate solution $u_{n}=\mathbb{P}_{n} u$ of equation (2.2) converges to its exact solution $u$ with not less than the second order convergence.

By Theorem 3 and (4.4), (4.5), we get

$$
\begin{aligned}
& \left|u-u_{n}\right| \leq M_{0} h^{2}, \\
& \left|u^{\prime}-u_{n}^{\prime}\right| \leq C h^{2}\|f\|_{W_{1}}\left\|\partial_{t} R_{t}\right\|_{W_{5}^{0}} \leq M_{1} h^{2}, \\
& \left|u^{\prime \prime}-u_{n}^{\prime \prime}\right| \leq C h\|f\|_{W_{1}}\left\|\partial_{t}^{2} R_{t}\right\|_{W_{5}^{0}} \leq M_{2} h .
\end{aligned}
$$

Furthermore, the following rate of convergence formulas can be obtained:

$$
r=\log _{2}\left(e_{h} / e_{\frac{h}{2}}\right)=2,
$$

where $e_{h}=M_{0} h^{2}$ is the error for $\left|u-u_{n}\right|, h=1 /(n-1)$.

\section{$5 \quad$ Numerical examples}

In this section, we give some computational results of some numerical experiments with methods based on preceding sections, to support our theoretical discussion.

Example 1. In order to focus on convergence order, we consider the following problem [5]

$$
\left\{\begin{array}{l}
u^{(4)}(x)-(1+c) u^{\prime \prime}(x)+c u(x)=\frac{1}{2} c x^{2}-1, \\
u(0)=1, u^{\prime}(0)=1, u^{\prime}(1)=1+\cosh (1), u(1)=3 / 2+\sinh (1),
\end{array}\right.
$$


where $c=10^{6}$, the exact solution is $u(x)=1+\frac{1}{2} x^{2}+\sinh (x)$. Table 1 lists the absolute pointwise error for $u-u_{n}$ and the rate of convergence $r$ to Example 1, using the present methods for various values of $n$, where $\bar{e}_{h}$ is the average error and $r_{i j}=\log _{2}\left(\frac{\bar{e}_{h_{i}}}{\bar{e}_{h_{j}}}\right)$. From Table 1, we confirm that our algorithm satisfies the formula (4.6) and (4.9). The left side of Figure 1 show the image of $u$, along with the image of $u_{n}$. Again we see the absolute error graph for first and second order derivative of solution in Figure 1, which follow from the formulas (4.7) and (4.8).

Table 1. The absolute pointwise error and the rate of convergence $r$ for Example 1

\begin{tabular}{|c|c|c|c|c|}
\hline$x$ & $h_{1}=\frac{1}{4}, n=5$ & $h_{2}=\frac{1}{8}, n=9$ & $h_{3}=\frac{1}{16}, n=17$ & $h_{4}=\frac{1}{32}, n=33$ \\
\hline 0 & $9.09 \mathrm{E}-11$ & $2.18 \mathrm{E}-11$ & $1.22 \mathrm{E}-10$ & $3.20 \mathrm{E}-11$ \\
\hline $1 / 4$ & $4.75 \mathrm{E}-8$ & $2.57 \mathrm{E}-8$ & $3.33 \mathrm{E}-9$ & $2.91 \mathrm{E}-10$ \\
\hline $1 / 2$ & $2.94 \mathrm{E}-6$ & $8.14 \mathrm{E}-8$ & $9.85 \mathrm{E}-9$ & $8.87 \mathrm{E}-10$ \\
\hline $3 / 4$ & $3.02 \mathrm{E}-6$ & $6.85 \mathrm{E}-8$ & $1.56 \mathrm{E}-8$ & $1.48 \mathrm{E}-10$ \\
\hline 1 & $2.36 \mathrm{E}-12$ & $9.42 \mathrm{E}-11$ & $1.91 \mathrm{E}-8$ & $4.08 \mathrm{E}-10$ \\
\hline $1 / 8$ & & $4.83 \mathrm{E}-8$ & $1.50 \mathrm{E}-12$ & $7.61 \mathrm{E}-10$ \\
\hline $3 / 8$ & & $5.30 \mathrm{E}-8$ & $6.71 \mathrm{E}-9$ & $2.01 \mathrm{E}-9$ \\
\hline $5 / 8$ & & $8.61 \mathrm{E}-8$ & $1.28 \mathrm{E}-8$ & $7.63 \mathrm{E}-10$ \\
\hline $7 / 8$ & & $1.30 \mathrm{E}-7$ & $1.88 \mathrm{E}-8$ & $3.91 \mathrm{E}-11$ \\
\hline $1 / 16$ & & & $2.67 \mathrm{E}-9$ & $2.61 \mathrm{E}-10$ \\
\hline $3 / 16$ & & & $1.43 \mathrm{E}-9$ & $3.69 \mathrm{E}-10$ \\
\hline $5 / 16$ & & & $5.01 \mathrm{E}-9$ & $4.02 \mathrm{E}-10$ \\
\hline $7 / 16$ & & & $8.34 \mathrm{E}-9$ & $1.58 \mathrm{E}-10$ \\
\hline $9 / 16$ & & & $1.13 \mathrm{E}-8$ & $2.27 \mathrm{E}-10$ \\
\hline $11 / 16$ & & & $1.42 \mathrm{E}-8$ & $5.17 \mathrm{E}-11$ \\
\hline $13 / 16$ & & & $1.75 \mathrm{E}-8$ & $6.90 \mathrm{E}-10$ \\
\hline $15 / 16$ & & & $2.21 \mathrm{E}-8$ & $4.64 \mathrm{E}-10$ \\
\hline $5 / 32$ & & & & $3.59 \mathrm{E}-9$ \\
\hline $7 / 32$ & & & & $1.63 \mathrm{E}-9$ \\
\hline $11 / 32$ & & & & $6.34 \mathrm{E}-10$ \\
\hline $15 / 32$ & & & & $9.65 \mathrm{E}-10$ \\
\hline $19 / 32$ & & & & $3.07 \mathrm{E}-9$ \\
\hline $31 / 32$ & & & & $5.62 \mathrm{E}-9$ \\
\hline $\bar{e}_{h}$ & $1.20 \mathrm{E}-6$ & $7.83 \mathrm{E}-8$ & $9.96 \mathrm{E}-9$ & $4.67 \mathrm{E}-10$ \\
\hline$r$ & $r_{12}=$ & $r_{2}$ & $=2.9$ & $4=3.1$ \\
\hline
\end{tabular}

Example 2. Consider the following linear fourth-order nonlocal problem [9]

$$
\left\{\begin{array}{l}
u^{(4)}(x)-e^{x} u^{\prime \prime \prime}(x)+u(x)=1-e^{x} \cosh (x)+2 \sinh (x), \\
u\left(\frac{1}{4}\right)=1+\sinh \left(\frac{1}{4}\right), \quad u^{\prime}\left(\frac{1}{4}\right)=\cosh \left(\frac{1}{4}\right), \\
u^{\prime \prime}\left(\frac{1}{4}\right)=\sinh \left(\frac{1}{4}\right), u\left(\frac{1}{2}\right)-u\left(\frac{3}{4}\right)=\sinh \left(\frac{1}{2}\right)-\sinh \left(\frac{3}{4}\right) .
\end{array}\right.
$$

The exact solution is $u(x)=1+\sinh (x)$. The numerical results are given in Table 2, where $r_{i j}=\log _{2}\left(\frac{e_{h_{i}}}{e_{h_{j}}}\right)$. From Table 2, we confirm that the numerical results justify the theoretical discussion. Comparison of the numerical results shows that the present method more accurate than the method in [9]. 


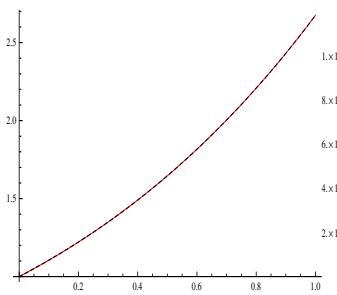

(a) $\mathrm{t}=1$

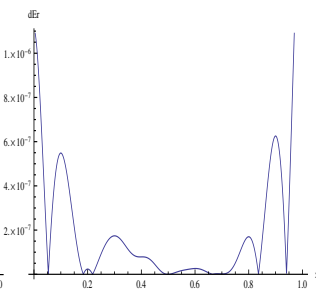

(b) $\mathrm{t}=5$

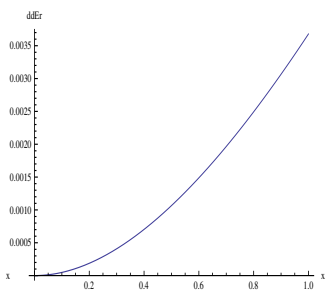

(c) $t=8$

Figure 1. Left side curves are the images of approximate solution $u_{33}(x)$ (dotted line)and exact solution $u(x)$ (solid line)for Example 1; Middle side curve is the absolute error between $u_{33}^{\prime}(x)$ and $u^{\prime}(x)$ for Example 1; Right side curve is the absolute error between $u_{33}^{\prime \prime}(x)$ and $u^{\prime \prime}(x)$ for Example 1

Table 2. The absolute pointwise error and the rate of convergence $r$ for Example 2

\begin{tabular}{lllllllll}
\hline$x$ & Method in[23] & $h_{1}=0.2$ & $h_{2}=0.1$ & $h_{3}=0.05$ & $h_{4}=0.025$ & $r_{12}$ & $r_{23}$ & $r_{34}$ \\
\hline 0 & $2.60 \mathrm{E}-3$ & $1.67 \mathrm{E}-4$ & $3.08 \mathrm{E}-5$ & $3.03 \mathrm{E}-6$ & $6.43 \mathrm{E}-7$ & 2.43 & 3.34 & 2.23 \\
0.1 & $4.70 \mathrm{E}-4$ & & $1.82 \mathrm{E}-5$ & $1.20 \mathrm{E}-6$ & $1.20 \mathrm{E}-7$ & & 3.90 & 3.32 \\
0.2 & $1.43 \mathrm{E}-5$ & \multirow{2}{*}{$1.50 \mathrm{E}-6$} & $3.20 \mathrm{E}-7$ & $2.77 \mathrm{E}-8$ & $3.77 \mathrm{E}-9$ & 2.14 & 2.53 & 2.87 \\
0.3 & $9.55 \mathrm{E}-6$ & & $9.87 \mathrm{E}-7$ & $1.81 \mathrm{E}-8$ & $2.81 \mathrm{E}-9$ & & 4.78 & 2.68 \\
0.4 & $1.83 \mathrm{E}-4$ & \multirow{4}{*}{$4.50 \mathrm{E}-5$} & $9.22 \mathrm{E}-6$ & $2.87 \mathrm{E}-7$ & $5.86 \mathrm{E}-8$ & 2.15 & 4.89 & 2.29 \\
0.5 & $5.77 \mathrm{E}-4$ & & $1.98 \mathrm{E}-6$ & $1.09 \mathrm{E}-7$ & $1.87 \mathrm{E}-8$ & & 4.18 & 2.61 \\
0.6 & $9.56 \mathrm{E}-4$ & $5.58 \mathrm{E}-4$ & $9.89 \mathrm{E}-5$ & $1.81 \mathrm{E}-6$ & $2.81 \mathrm{E}-7$ & 2.49 & 5.77 & 2.68 \\
0.7 & $9.14 \mathrm{E}-4$ & & $9.10 \mathrm{E}-5$ & $1.08 \mathrm{E}-6$ & $1.08 \mathrm{E}-7$ & & 6.39 & 3.32 \\
0.8 & $6.74 \mathrm{E}-5$ & $2.36 \mathrm{E}-3$ & $4.98 \mathrm{E}-5$ & $9.49 \mathrm{E}-6$ & $6.69 \mathrm{E}-7$ & 5.56 & 2.39 & 3.87 \\
0.9 & $2.63 \mathrm{E}-3$ & & $2.33 \mathrm{E}-4$ & $1.97 \mathrm{E}-5$ & $2.61 \mathrm{E}-6$ & & 3.54 & 2.91 \\
1 & $7.57 \mathrm{E}-3$ & $6.67 \mathrm{E}-3$ & $4.08 \mathrm{E}-4$ & $3.84 \mathrm{E}-5$ & $8.11 \mathrm{E}-6$ & 4.02 & 3.40 & 2.24 \\
\hline
\end{tabular}

Example 3. Consider the following linear third-order three points boundary value problem [4].

$$
\left\{\begin{array}{l}
u^{\prime \prime \prime}(x)-\alpha u^{\prime \prime}(x)-\beta u^{\prime}(x)+\gamma u(x)=f(x), \\
u(1)=u(-1)=u^{\prime}(1)=0
\end{array}\right.
$$

where $f(x)$ is chosen such that the exact solution for Equation (5.2) is $u(x)=$ $\left(1-x^{2}\right) x^{j} \sin (m \pi x), j, m \in N$.

Table 3. Maximum absolute errors and the rate of convergence for Example 3

\begin{tabular}{lllllllll}
\hline$j$ & $m$ & $\alpha$ & $\beta$ & $\gamma$ & Method in $[23]$ & Our method $n=8$ & Our method $n=16$ & $\mathrm{r}$ \\
\hline 1 & 1 & 0 & 0 & 0 & $2.558 \mathrm{E}-3$ & $7.725 \mathrm{E}-4$ & $1.723 \mathrm{E}-4$ & 2.16 \\
0 & 1 & 2 & 3 & 4 & $4.472 \mathrm{E}-3$ & $3.099 \mathrm{E}-4$ & $7.017 \mathrm{E}-5$ & 2.10 \\
1 & 2 & 0 & 1 & 0 & $1.119 \mathrm{E}-1$ & $3.860 \mathrm{E}-2$ & $1.001 \mathrm{E}-2$ & 1.98 \\
2 & 1 & 1 & 0 & 1 & $1.578 \mathrm{E}-2$ & $8.341 \mathrm{E}-3$ & $3.804 \mathrm{E}-4$ & 2.05 \\
\hline
\end{tabular}

In Table 3, the maximum errors $\mathrm{E}$ for $u-u_{n}$ and the rate of convergence $r$ to equation (5.2) are listed, using our present algorithm for various values 
of $j, m$ and the coefficients $\alpha, \beta$ and $\gamma$. Furthermore, we give a comparison between our algorithm and the method in [4].

\section{Conclusions}

To summarize, in this paper, we strictly present convergence order on the basis of previous research. In the reproducing kernel space, some convergent theorems and error order estimation are given for the first time. On the assumption of equation (2.2), we get that the approximate solution has no less than the second-order convergence. The numerical results show that the present method is an accurate and reliable analytical technique. It is worthy to note that the approach detailed here can be readily adapted to the case of linear integral and integro-differential equation with various boundary value conditions.

\section{References}

[1] K. Atkinson and W. Han. Theoretical Numerical Analysis. Texts in Applied Mathematics. Springer-Verlag, New York, 2009. http://dx.doi.org/10.1007/9781-4419-0458-4.

[2] Z. Chen and Y. Lin. The exact solution of a linear integral equation with weakly singular kernel. Journal of Mathematical Analysis and Applications, 344(2):726734, 2008. http://dx.doi.org/10.1016/j.jmaa.2008.03.023.

[3] D.-Q. Dai and Y. Huang. Nonlocal boundary problems for a thirdorder one-dimensional nonlinear pseudoparabolic equation. Nonlinear Analysis: Theory, Methods \& Applications, 66(1):179-191, 2007. http://dx.doi.org/10.1016/j.na.2005.11.021.

[4] E.H. Doha, W.M. Abd-Elhameed and Y.H. Youssri. New algorithms for solving third- and fifth-order two point boundary value problems based on nonsymmetric generalized Jacobi method. Journal of Advanced Research, 6(5):673-686, 2015. http://dx.doi.org/10.1016/j.jare.2014.03.003.

[5] F. Geng. A new reproducing kernel Hilbert space method for solving nonlinear fourth-order boundary value problems. Applied Mathematics and Computation, 213(1):163-169, 2009. http://dx.doi.org/10.1016/j.amc.2009.02.053.

[6] F. Geng. Solving singular second order three-point boundary value problems using reproducing kernel Hilbert space method. Applied Mathematics and Computation, 215(6):2095-2102, 2009. http://dx.doi.org/10.1016/j.amc.2009.08.002.

[7] F. Geng. A novel method for solving a class of singularly perturbed boundary value problems based on reproducing kernel method. Applied Mathematics and Computation, 218(8):4211-4215, 2011. http://dx.doi.org/10.1016/j.amc.2011.09.052.

[8] F. Geng and M. Cui. A reproducing kernel method for solving nonlocal fractional boundary value problems. Applied Mathematics Letters, 25(5):818-823, 2012. http://dx.doi.org/10.1016/j.aml.2011.10.025.

[9] Y. Lin and J. Lin. A numerical algorithm for solving a class of linear nonlocal boundary value problems. Applied Mathematics Letters, 23(9):997-1002, 2010. http://dx.doi.org/10.1016/j.aml.2010.04.025. 
[10] Y. Lin and J. Lin. Numerical method for solving the nonlinear four-point boundary value problems. Communications Nonlinear Science and Numerical Simulation, 15(12):3855-3864, 2010. http://dx.doi.org/10.1016/j.cnsns.2010.02.013.

[11] Y. Lin, J. Niu and M. Cui. A numerical solution to nonlinear second order three-point boundary value problems in the reproducing kernel space. Applied Mathematics and Computation, 218(14):7362-7368, 2012. http://dx.doi.org/10.1016/j.amc.2011.11.009.

[12] L. López, E.P Sinusía and N.M. Temme. Multi-point Taylor approximations in one-dimensional linear boundary value problems. Applied Mathematics and Computation, 207(2):519-527, 2009. http://dx.doi.org/10.1016/j.amc.2008.11.015.

[13] M. Mohammadi and R. Mokhtari. Solving the generalized regularized long wave equation on the basis of a reproducing kernel space. Journal of Computational and Applied Mathematics, 235(14):4003-4014, 2011. http://dx.doi.org/10.1016/j.cam.2011.02.012.

[14] M. Mohammadi and R. Mokhtari. A new algorithm for solving one-dimensional Schrödinger equation in the reproducing kernel space. Iranian Journal of Science E Technology, Transaction A: Science, 37:513-526, 2013.

[15] M. Mohammadi and R. Mokhtari. A reproducing kernel method for solving a class of nonlinear systems of PDEs. Mathematical Modelling and Analysis, 19(2):180-198, 2014. http://dx.doi.org/10.3846/13926292.2014.909897.

[16] M. Mohammadi, R. Mokhtari and H. Panahipour. Solving two parabolic inverse problems with a nonlocal boundary condition in the reproducing kernel space. Applied and Computational Mathematics, 13(1):91-106, 2014.

[17] R. Mokhtari, F. Toutian Isfahani and M. Mohammadi. Reproducing kernel method for solving nonlinear differential-difference equations. Abstract and Applied Analysis, 2012:1-8, 2012. http://dx.doi.org/10.1155/2012/514103.

[18] J. Niu and P. Li. Numerical algorithm for the third-order partial differential equation with three-point boundary value problem. Abstract and Applied Analysis, 2014:1-7, 2014. http://dx.doi.org/10.1155/2014/630671.

[19] J. Niu, Y.Z. Lin and C.P. Zhang. Approximate solution of nonlinear multi-point boundary value problem on the half-line. Mathematical Modelling and Analysis, 17(2):190-202, 2012. http://dx.doi.org/10.3846/13926292.2012.660889.

[20] J. Niu, Y.Z. Lin and C.P. Zhang. Numerical solution of nonlinear three-point boundary value problem on the positive half-line. Mathematical Methods in the Applied Sciences, 35(13):1601-1610, 2012. http://dx.doi.org/10.1002/mma.2549.

[21] B.Y. Wu and Y.Z. Lin. Applied Reproducing Kernel Space. Science Press, Beijing, China, 2012.

[22] H. Yao and Y. Lin. Solving singular boundary-value problems of higher evenorder. Journal of Computational and Applied Mathematics, 223(2):703-713, 2009. http://dx.doi.org/10.1016/j.cam.2008.02.010.

[23] R. Zhang and Y. Lin. A novel method for nonlinear boundary value problems. Journal of Computational and Applied Mathematics, 282:77-82, 2015. http://dx.doi.org/10.1016/j.cam.2014.12.035.

[24] Y.F. Zhou, M.G. Cui and Y.Z. Lin. A computational method for nonlinear $2 m$-th order boundary value problems. Mathematical Modelling and Analysis, 15(4):571-586, 2010. http://dx.doi.org/10.3846/1392-6292.2010.15.571-586. 